\title{
Approximation of functions by Bernoulli wavelet and its applications in solution of Volterra integral equation of second kind
}

Received: 12 February 2021 / Accepted: 1 November 2021 / Published online: 2 December 2021

(C) The Author(s) 2021

\begin{abstract}
In this paper, two new estimators $E_{2^{k-1}, 0}^{(1)}(f)$ and $E_{2^{k-1}, M}^{(1)}(f)$ of characteristic function and an estimator $E_{2^{k-1}, M}^{(2)}(f)$ of function of Hölder's class $H^{\alpha}[0,1)$ of order $0<\alpha \leqslant 1$ have been established using Bernoulli wavelets. A new technique has been applied for solving Volterra integral equation of second kind using Bernoulli wavelet operational matrix of integration as well as product operational matrix. These matrices have been utilized to reduce the Volterra integral equation into a system of algebraic equations, which are easily solvable. Some examples are illustrated to show the validity and efficiency of proposed technique of this research paper.
\end{abstract}

Mathematics Subject Classification $\quad 42 \mathrm{C} 40 \cdot 65 \mathrm{~T} 60 \cdot 45 \mathrm{D} 05$

\section{Introduction}

Wavelet analysis is recent and advanced analysis in pure as well as applied mathematical research area. It has been applied in different branches of science and technology such as engineering, image processing, signal analysis, time frequency analysis, and fast numerical algorithms. There are several problems of science and technology in the form of integral equations. There are various types of methods to find the numerical solution of integral equations. Sometimes it is difficult to find the solution of the integral equations analytically. Therefore, there is need of approximate solution.

Several researchers such as Liang [6], Maleknejad [8], Keshvarz [3,13] and Shah [14,19] have been solving integral equations using wavelet methods. Lepik [7] and Yousefi [20] have presented numerical methods to solve the Fredholm integral equation by Haar and CAS wavelet method. Shah and Irfan $[15,16,18]$ have used wavelet in bio-heat transfer model and population growth model.

Bernoulli wavelets have been applied to solve fractional order differential equations and calculus of variation problems by Keshavarz [3,4]. We have presented Bernoulli wavelet method to solve linear Volterra integral equations of the form:

$$
y(x)=f(x)+\int_{0}^{x} K(x, t) y(t) \mathrm{d} t
$$

S. Lal · S. Kumar $(\varangle)$

Department of Mathematics, Institute of Science, Banaras Hindu University, Varanasi 221005, India

E-mail: satishkumar3102@gmail.com

S. Lal

E-mail: shyam_lal@rediffmail.com 
and nonlinear Volterra integral equation of the form:

$$
y(x)=f(x)+\int_{0}^{x} K(x, t) F(y(t)) \mathrm{d} t
$$

where $f \in L^{2}[0,1)$ and $K \in L^{2}[0,1) \times L^{2}[0,1)$ are known functions, $F$ is a function of $y$ and $y$ is unknown function to be determined [1,9-11].

The main objectives of this research paper are following:

1. To establish estimation of characteristic function and function of Hölder's class $H^{\alpha}[0,1)$ of order $0<\alpha \leqslant 1$.

2. To present a numerical method for solving linear Volterra integral equation (1.1) and nonlinear Volterra integral equation (1.2).

3. To compare the Bernoulli wavelet solution of integral equation (1.1) and (1.2) with its exact solution.

It is remarkable that the solution of (1.1) and (1.2) obtained by Bernoulli wavelet technique and its exact solution are almost same.

\section{Definitions and preliminaries}

\subsection{Bernoulli wavelets}

Bernoulli wavelets $B_{n, m}$ have four arguments: $\hat{n}=n-1, n=1,2, \ldots, 2^{k-1}$, where $k$ is some positive integer, $m$ is the order of Bernoulli polynomials and $t$ is the normalized time. Then, $B_{n, m}$ are defined on the interval $[0,1)$ as follows:

$$
B_{n, m}(t)= \begin{cases}2^{\frac{k-1}{2}} \tilde{\beta_{m}}\left(2^{k-1} t-\hat{n}\right), & \text { if } \frac{\hat{n}}{2^{k-1}} \leqslant t<\frac{\hat{n}+1}{2^{k-1}} \\ 0, & \text { otherwise }\end{cases}
$$

where

$$
\tilde{\beta}_{m}(t)= \begin{cases}1, & \text { if } m=0 \\ \frac{1}{\sqrt{\frac{(-1)^{m-1}(m ! !)^{2}}{(2 m) !} \alpha_{2 m}}} \beta_{m}(t), & \text { if } m>0,\end{cases}
$$

for $m=0,1,2, \ldots, M-1$, and $n=1,2, \ldots, 2^{k-1}$. The coefficient $\frac{1}{\sqrt{\frac{(-1)^{m-1}(m !)^{2}}{(2 m) !} \alpha_{2 m}}}$ is for normality, $\beta_{m}(t)$ is Bernoulli polynomial of order $m$, which is given by

$$
\beta_{m}(t)=\sum_{i=0}^{m}\left(\begin{array}{c}
m \\
i
\end{array}\right) \alpha_{m-i} t^{i}
$$

$\alpha_{j}$ are Bernoulli numbers and can be defined by the identity $\frac{t}{e^{t}-1}=\sum_{i=0}^{\infty} \alpha_{i} \frac{t^{i}}{i !}$. The first few Bernoulli numbers are $\alpha_{0}=1, \alpha_{1}=\frac{-1}{2}, \alpha_{2}=\frac{1}{6}, \alpha_{4}=\frac{-1}{30}, \alpha_{6}=\frac{1}{42}, \alpha_{8}=\frac{-1}{30}, \alpha_{10}=\frac{5}{66}, \ldots$ and $\alpha_{2 i+1}=0, i=$ $1,2,3, \ldots$

The first few Bernoulli polynomials are

$\beta_{0}(t)=1, \quad \beta_{1}(t)=t-\frac{1}{2}, \quad \beta_{2}(t)=t^{2}-t+\frac{1}{6}$,

$\beta_{3}(t)=t^{3}-\frac{3}{2} t^{2}+\frac{1}{2} t, \quad \beta_{4}(t)=t^{4}-2 t^{3}+t^{2}-\frac{1}{30}$,

$\beta_{5}(t)=t^{5}-\frac{5}{2} t^{4}+\frac{5}{3} t^{3}-\frac{1}{6} t, \quad \beta_{6}(t)=t^{6}-3 t^{5}+\frac{5}{2} t^{4}-\frac{1}{2} t^{2}+\frac{1}{42}$, (Shiralashetti) [11,12,17].

2.2 Properties of Bernoulli polynomial $[2,5]$
1. $\beta_{m}^{\prime}(t)=m \beta_{m-1}(t)$
2. $\int_{0}^{1}\left|\beta_{m}(t)\right| \mathrm{d} t<16 \frac{m !}{(2 \pi)^{m+1}}$
3. $\beta_{m}(0)=(-1)^{m} \beta_{m}(1)$
4. $\int_{0}^{1} \beta_{m}(x) \mathrm{d} x=0$ 


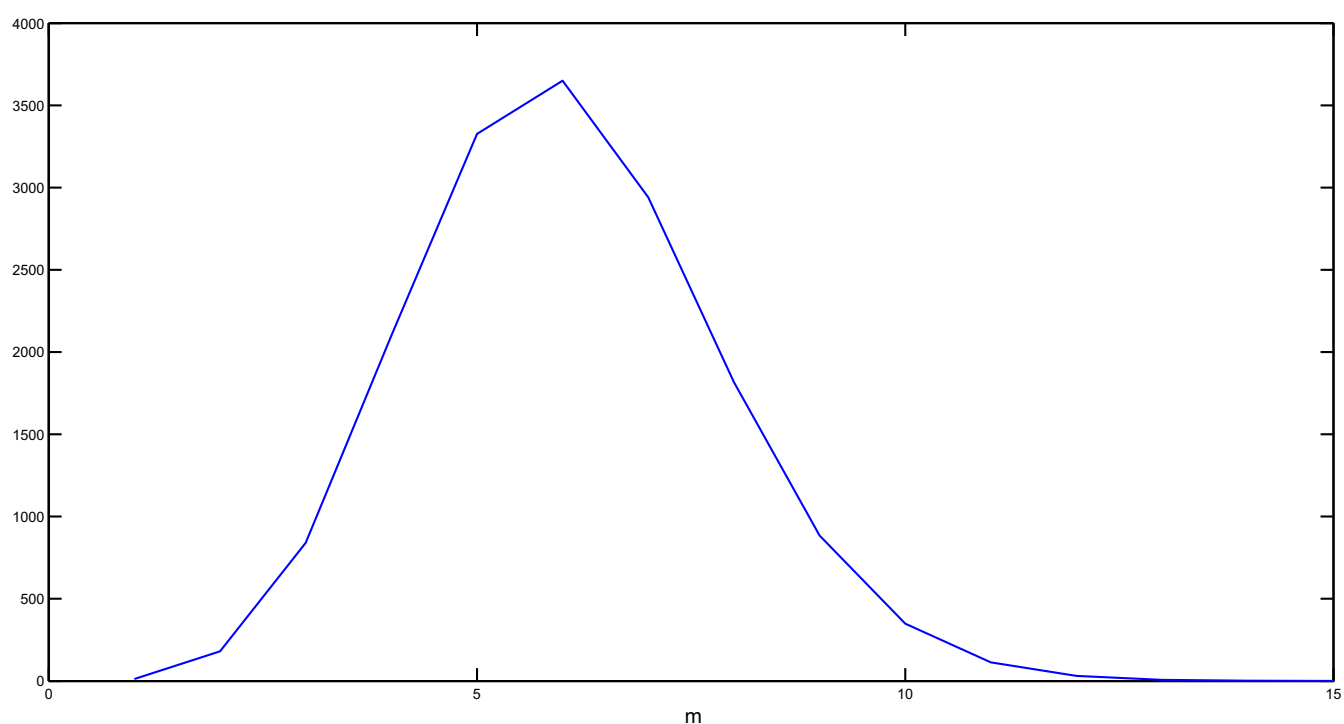

Fig. 1 Graph between $\mathrm{m}$ and normality factor.

2.3 Graph between $m$ and the factor $\left|\frac{1}{\frac{(-1)^{m-1}(m !)^{2}}{(2 m) !} \alpha_{2 m}}\right|$

2.4 Function of Hölder's class $H^{\alpha}[0,1)$

A function $f$ is of Hölder's class $H^{\alpha}[0,1)$ of order $0<\alpha \leqslant 1$ if $f$ satisfies the following condition:

$$
|f(x)-f(y)| \leqslant A|x-y|^{\alpha}, \quad \forall x, y \in \mathbb{R}
$$

for some positive constant $A$ (Zheng and Wei [21]).

\section{Approximation of function}

A function $f \in L^{2}[0,1)$ can be expanded as

$$
f(t)=\sum_{n=1}^{\infty} \sum_{m=0}^{\infty} c_{n, m} B_{n, m}(t)
$$

where the coefficients $c_{n, m}$ are given by

$$
c_{n, m}=<f, B_{n, m}>
$$

where $<,>$ represents inner product in $L^{2}[0,1)$.

$\left(2^{k-1}, M\right)$ th partial sum $S_{2^{k-1}, M}(f)(t)$ of infinite series (3.1) is given by

$$
S_{2^{k-1}, M}(f)(t)=\sum_{n=1}^{2^{k-1}} \sum_{m=0}^{M-1} c_{n, m} B_{n, m}(t)=C^{T} B(t)
$$

where $C$ and $B(t)$ are given by

$$
C=\left[c_{1,0}, c_{1,1}, \ldots, c_{1, M-1}, c_{2,0}, \ldots, c_{2, M-1}, \ldots, c_{2^{k}, 0}, \ldots, c_{2^{k-1}, M-1}\right]^{T}=\left[c_{1}, c_{2, \ldots, c_{2^{k-1} M}}\right]^{T}
$$




$$
\begin{aligned}
B(t) & =\left[B_{1,0}(t), B_{1,1}(t), \ldots, B_{1, M-1}(t), B_{2,0}(t), \ldots, B_{2, M-1}(t), \ldots, B_{2^{k}, 0}(t), \ldots, B_{2^{k-1}, M-1}(t)\right]^{T} \\
& =\left[B_{1}(t), B_{2}(t), \ldots, B_{2^{k-1} M}(t)\right]^{T} .
\end{aligned}
$$

Now $K(x, t) \in L^{2}[0,1) \times L^{2}[0,1)$ can be expanded as

$$
K(x, t)=B^{T}(x) \mathbf{K} B(t)
$$

where $\mathbf{K}$ is $2^{k-1} M \times 2^{k-1} M$ matrix with entries $\mathbf{K}_{i, j}=<B_{i}(x),<K(x, t), B_{j}(t)>>$. For $k=3$ and $M=3$, 12 Bernoulli wavelet basis functions are given by

$$
\begin{aligned}
& B_{1,0}(t)=2 \\
& \left.\begin{array}{l}
B_{1,1}(t)=2 \sqrt{3}(8 t-1) \\
B_{1,2}(t)=2 \sqrt{5}\left(96 t^{2}-24 t+1\right)
\end{array}\right\} \quad 0 \leqslant t<\frac{1}{4}, \\
& B_{2,0}(t)=2 \\
& \left.\begin{array}{l}
B_{2,1}(t)=2 \sqrt{3}(8 t-3) \\
B_{2,2}(t)=2 \sqrt{5}\left(96 t^{2}-72 t+13\right)
\end{array}\right\} \quad \frac{1}{4} \leqslant t<\frac{1}{2}, \\
& B_{3,0}(t)=2 \\
& B_{3,1}(t)=2 \sqrt{3}(8 t-5) \\
& \left.B_{3,2}(t)=2 \sqrt{5}\left(96 t^{2}-120 t+37\right)\right) \\
& \frac{1}{2} \leqslant t<\frac{3}{4}, \\
& B_{4,0}(t)=2 \\
& B_{4,1}(t)=2 \sqrt{3}(8 t-7) \\
& B_{4,2}(t)=2 \sqrt{5}\left(96 t^{2}-168 t+73\right) \\
& \frac{3}{4} \leqslant t<1 .
\end{aligned}
$$

\subsection{Bernoulli wavelets operational matrix of integration}

For $k=3$ and $M=3$, the $2^{k-1} M \times 2^{k-1} M$ Bernoulli wavelet operational matrix of integration given by Razzaghi [4] as follows:

$$
\mathbf{P}=\frac{1}{4}\left[\begin{array}{cccccccccccc}
\frac{1}{2} & \frac{1}{2 \sqrt{3}} & 0 & 1 & 0 & 0 & 1 & 0 & 0 & 1 & 0 & 0 \\
-\frac{1}{2 \sqrt{3}} & 0 & \frac{1}{2 \sqrt{15}} & 0 & 0 & 0 & 0 & 0 & 0 & 0 & 0 & 0 \\
0 & 0 & 0 & 0 & 0 & 0 & 0 & 0 & 0 & 0 & 0 & 0 \\
0 & 0 & 0 & \frac{1}{2} & \frac{1}{2 \sqrt{3}} & 0 & 1 & 0 & 0 & 1 & 0 & 0 \\
0 & 0 & 0 & -\frac{1}{2 \sqrt{3}} & 0 & \frac{1}{2 \sqrt{15}} & 0 & 0 & 0 & 0 & 0 & 0 \\
0 & 0 & 0 & 0 & 0 & 0 & 0 & 0 & 0 & 0 & 0 & 0 \\
0 & 0 & 0 & 0 & 0 & 0 & \frac{1}{2} & \frac{1}{2 \sqrt{3}} & 0 & 1 & 0 & 0 \\
0 & 0 & 0 & 0 & 0 & 0 & -\frac{1}{2 \sqrt{3}} & 0 & \frac{1}{2 \sqrt{15}} & 0 & 0 & 0 \\
0 & 0 & 0 & 0 & 0 & 0 & 0 & 0 & 0 & 0 & 0 & 0 \\
0 & 0 & 0 & 0 & 0 & 0 & 0 & 0 & 0 & \frac{1}{2} & \frac{1}{2 \sqrt{3}} & 0 \\
0 & 0 & 0 & 0 & 0 & 0 & 0 & 0 & 0 & -\frac{1}{2 \sqrt{3}} & 0 & \frac{1}{2 \sqrt{15}} \\
0 & 0 & 0 & 0 & 0 & 0 & 0 & 0 & 0 & 0 & 0 & 0
\end{array}\right]
$$

\subsection{Bernoulli wavelets operational matrix of product}

The product of two Bernoulli wavelet functions is given by $C^{T} B(t) B(t)^{T} \approx B(t) \tilde{C}$, where $\tilde{C}$ is a $2^{k-1} M \times$ $2^{k-1} M$ product operational matrix. The entries of $B(t) B(t)^{T}$ are given by $B_{i j} B_{r s}=0$ for $i \neq r, B_{i 0} B_{i j}=$ $2 B_{i j}$, 
$B_{i 1} B_{i 1}=2 B_{i 0}+\frac{4}{\sqrt{5}} B_{i 2}, \quad B_{i 1} B_{i 2}=\frac{4}{\sqrt{5}} B_{i 1}, \quad B_{i 2} B_{i 2}=2 B_{i 0}+\frac{4 \sqrt{5}}{7} B_{i 2}$.

Using these, we get

$$
\tilde{C}=\left[\begin{array}{cccc}
\tilde{C}_{1} & 0 & 0 & 0 \\
0 & \tilde{C}_{2} & 0 & 0 \\
0 & 0 & \tilde{C}_{3} & 0 \\
0 & 0 & 0 & \tilde{C}_{4}
\end{array}\right] \text { where } \quad \tilde{C}_{i}=\left[\begin{array}{ccc}
2 c_{i 0} & 2 c_{i 1} & 2 c_{i 2} \\
2 c_{i 1} & 2 c_{i 0}+\frac{4}{\sqrt{5}} c_{i 2} & \frac{4}{\sqrt{5}} c_{i 1} \\
2 c_{i 2} & \frac{4}{\sqrt{5}} c_{i 1} & 2 c_{i 0}+\frac{4 \sqrt{5}}{7} c_{i 2}
\end{array}\right] \text { for } i=1,2,3,4 .
$$

\section{Results}

Next, we prove the following Theorems:

Theorem 4.1 Let $f(x)=x \chi_{\left[\frac{n_{1}-1}{2^{k-1}}, \frac{n_{1}}{2^{k-1}}\right)}$ with $1<n_{1}<2^{k-1}$ such that its Bernoulli wavelet expansion is given by series

$$
f(x)=\sum_{n=1}^{\infty} \sum_{m=0}^{\infty} c_{n, m} B_{n, m}(x),
$$

and $\left(2^{k-1}, M\right)$ th partial sum $S_{2^{k-1}, M}(f)$ given by

$$
S_{2^{k-1}, M}(f)(x)=\sum_{n=1}^{2^{k-1}} \sum_{m=0}^{M-1} c_{n, m} B_{n, m}(x) .
$$

Then approximation error $E_{2^{k-1}, M}^{(1)}(f)$ of $f$ by $S_{2^{k-1}, M}(f)$ is given by

$$
E_{2^{k-1}, M}^{(1)}(f)= \begin{cases}o\left(\frac{1}{2^{\frac{1}{2}(k-1)}}\right), & \text { if } M=0 \\ o\left(\frac{1}{2^{\frac{3}{2}(k-1)} \sqrt{M+1}}\right), & \text { if } M \geq 1 .\end{cases}
$$

Theorem 4.2 Let $f \in L^{2}[0,1)$ be a function of Hölder's class $H^{\alpha}[0,1)$ of order $0<\alpha \leqslant 1$, such that its Bernoulli wavelet expansion is given by the series (4.1) with $\left(2^{k-1}, M\right)$ th partial sum $S_{2^{k-1}, M}(f)$. Then approximation error $E_{2^{k-1}, M}^{(2)}(f)$ of $f$ by $S_{2^{k-1}, M}(f)$ is given by

$$
E_{2^{k-1}, M}^{(2)}(f)=o\left(\frac{1}{\sqrt{M+1} 2^{(k-1) \alpha}}\right) .
$$

To prove the above theorems, first we prove the following Lemma:

Lemma 4.1 If $f \in L^{2}[0,1)$ is a function such that its Bernoulli wavelet expansion is given by the series (4.1) with $\left(2^{k-1}, M\right)^{\text {th }}$ partial sum $S_{2^{k-1}, M}(f)(x)$ given by (4.2), then

$$
\left\|f-S_{2^{k-1}, M}(f)\right\|_{2}^{2}=\sum_{n=1}^{2^{k-1}} \sum_{m=M}^{\infty}\left|c_{n, m}\right|^{2}
$$

Proof

$$
\begin{aligned}
f(x)-S_{2^{k-1}, M}(f)(x) & =\sum_{n=1}^{\infty} \sum_{m=0}^{\infty} c_{n, m} B_{n, m}(x)-\sum_{n=1}^{2^{k-1}} \sum_{m=0}^{M-1} c_{n, m} B_{n, m}(x) \\
& =\sum_{n=1}^{2^{k-1}}\left(\sum_{m=0}^{M-1}+\sum_{m=M}^{\infty}\right) c_{n, m} B_{n, m}(x)-\sum_{n=1}^{2^{k-1}} \sum_{m=0}^{M-1} c_{n, m} B_{n, m}(x), \quad\left(\operatorname{by~def}^{n} \text { of } B_{n, m}\right)
\end{aligned}
$$




$$
\begin{aligned}
& =\sum_{n=1}^{2^{k-1}} \sum_{m=0}^{M-1} c_{n, m} B_{n, m}(x)+\sum_{n=1}^{2^{k-1}} \sum_{m=M}^{\infty} c_{n, m} B_{n, m}(x)-\sum_{n=1}^{2^{k-1}} \sum_{m=0}^{M-1} c_{n, m} B_{n, m}(x) \\
& =\sum_{n=1}^{2^{k-1}} \sum_{m=M}^{\infty} c_{n, m} B_{n, m}(x) \\
& \left(f(x)-S_{2^{k-1}, M}(f)(x)\right)^{2}=\sum_{n=1}^{2^{k-1}} \sum_{m=M}^{\infty} c_{n, m}^{2} B_{n, m}^{2}(x)+2 \sum_{1 \leqslant n \neq n^{\prime} \leq 2^{k-1}} \sum_{M \leq m \neq m^{\prime}<\infty} \sum_{n, m} c_{n^{\prime}, m^{\prime}} B_{n, m}^{T}(x) B_{n^{\prime}, m^{\prime}}(x) \\
& \left\|f-S_{2^{k-1}, M}(f)\right\|_{2}^{2}=\int_{0}^{1}\left|f(x)-S_{2^{k-1}, M}(f)(x)\right|^{2} \mathrm{~d} x \\
& \leqslant \sum_{n=1}^{2^{k-1}} \sum_{m=M}^{\infty}\left|c_{n, m}\right|^{2} \int_{0}^{1}\left|B_{n, m}(x)\right|^{2} \mathrm{~d} x+2 \sum_{1 \leqslant n \neq n^{\prime} \leq 2^{k-1}} \sum_{M \leq m \neq m^{\prime}<\infty} \sum_{n, m}\left|c_{n, m}\right|\left|c_{n^{\prime}, m^{\prime}}\right| \\
& \int_{0}^{1}\left|B_{n, m}^{T}(x) B_{n^{\prime}, m^{\prime}}(x)\right| \mathrm{d} t=\sum_{n=1}^{2^{k-1}} \sum_{m=M}^{\infty}\left|c_{n, m}\right|^{2} \text {, by orthonormality of }\left\{B_{n, m}\right\} \text {. }
\end{aligned}
$$

Proof of Theorem 4.1 From (3.2), $\quad c_{n, m}=<f(x), B_{n, m}(x)>$

Case 1: $m=0$

$$
\begin{aligned}
c_{n_{1}, 0}= & \int_{\frac{n-1}{2^{k-1}}}^{\frac{n}{2^{k-1}}} \times \chi_{\left[\frac{n_{1}-1}{2^{k-1}}, \frac{n_{1}}{2^{k-1}}\right)} B_{n_{1}, 0}(x) \mathrm{d} x \\
= & \int_{\frac{n_{1}-1}{2^{k-1}}}^{\frac{n_{1}}{2^{k-1}}} \times 2^{\frac{k-1}{2}} \tilde{\beta_{0}}\left(2^{k-1} x-n_{1}+1\right) \mathrm{d} x \\
= & 2^{\frac{-3}{2}(k-1)} \int_{0}^{1}\left(u+n_{1}-1\right) \tilde{\beta}_{0}(u) \mathrm{d} u, \quad\left(\text { putting } 2^{k-1} x-n_{1}+1=u\right) \\
= & 2^{\frac{-3}{2}(k-1)} \int_{0}^{1}\left(u+n_{1}-1\right) \mathrm{d} u=2^{\frac{-3}{2}(k-1)}\left(n_{1}-\frac{1}{2}\right) \leqslant 2^{\frac{-1}{2}(k-1)}, \quad\left(\because 1<n_{1}<2^{k-1}\right) . \\
& \left\|f-S_{2^{k-1}, 0}(f)\right\|_{2}^{2}=\left|c_{n_{1}, 0}\right|^{2} \leq 2^{-(k-1)} \quad(\text { by Lemma 4.1) } \\
& E_{2^{k-1}, 0}^{(1)}(f)=\left\|f-S_{2^{k-1}, 0}(f)\right\|_{2}=o\left(\frac{1}{2^{\frac{1}{2}(k-1)}}\right) .
\end{aligned}
$$

Case 2: $m=M \geq 1$

$$
\begin{aligned}
c_{n_{1}, m} & =\int_{\frac{n-1}{2^{k-1}}}^{\frac{n}{2^{k-1}}} x \chi_{\left[\frac{n_{1}-1}{2^{k-1}}, \frac{n_{1}}{2^{k-1}}\right)} B_{n_{1}, m}(x) \mathrm{d} x \\
& =\int_{\frac{n_{1}-1}{2^{k-1}}}^{\frac{2_{1}}{2^{k-1}}} x 2^{\frac{k-1}{2}} \tilde{\beta_{m}}\left(2^{k-1} x-n_{1}+1\right) \mathrm{d} x \\
& =2^{\frac{-3}{2}(k-1)} \frac{1}{\sqrt{\frac{(-1)^{m-1}(m !)^{2}}{(2 m) !} \alpha_{2 m}}} \int_{0}^{1}\left(u+n_{1}-1\right) \beta_{m}(u) \mathrm{d} u, \quad\left(\text { putting } 2^{k-1} x-n_{1}+1=u\right) \\
& =2^{\frac{-3}{2}(k-1)} \frac{1}{\sqrt{\frac{(-1)^{m-1}(m !)^{2}}{(2 m) !} \alpha_{2 m}}} \int_{0}^{1}\left(u+n_{1}-1\right) \frac{1}{m+1} \frac{\mathrm{d}}{\mathrm{d} u}\left(\beta_{m+1}(u)\right) \mathrm{d} u, \quad \text { (by property (1)) } \\
& =2^{\frac{-3}{2}(k-1)} \frac{1}{\sqrt{\frac{(-1)^{m-1}(m !)^{2}}{(2 m) !} \alpha_{2 m}}} \frac{m+1}{\left.m+n_{1} \beta_{m+1}(1)-\left(n_{1}-1\right) \beta_{m+1}(0)\right], \quad \text { (integrating by part) }}
\end{aligned}
$$




$$
\begin{gathered}
=\frac{2^{\frac{-3}{2}(k-1)}}{\sqrt{\frac{(-1)^{m-1}(m !)^{2}}{(2 m) !} \alpha_{2 m}}} \frac{1}{m+1} \beta_{m+1}(0) \leq 2^{\frac{-3}{2}(k-1)} \frac{1}{\sqrt{\frac{(-1)^{m-1}(m !)^{2}}{(2 m) !} \alpha_{2 m}}} \frac{1}{m+1}, \quad\left(\because \beta_{m}(1)=\beta_{m}(0)\right) \\
\left\|f-S_{2^{k-1}, M}(f)\right\|_{2}^{2}=\sum_{m=M}^{\infty}\left|c_{n_{1}, m}\right|^{2}=\sum_{m=M}^{\infty} 2^{-3(k-1)}\left|\frac{1}{\frac{(-1)^{m-1}(m !)^{2}}{(2 m) !} \alpha_{2 m}}\right| \frac{1}{(m+1)^{2}} . \quad \text { (by Lemma 4.1) }
\end{gathered}
$$

From the graph (2.3), we have $\left|\frac{1}{\frac{(-1)^{m-1}(m !)^{2}}{(2 m) !} \alpha_{2 m}}\right| \leq 3650$

$$
\begin{aligned}
\left\|f-S_{2^{k-1}, M}(f)\right\|_{2}^{2} & \leq 3650\left(2^{-3(k-1)}\right) \int_{x=M}^{\infty} \frac{1}{(x+1)^{2}} \mathrm{~d} x=o\left(\frac{1}{\left(2^{3(k-1)}\right)(M+1)}\right) \\
E_{2^{k-1}, M}^{(1)}(f) & =\left\|f-S_{2^{k-1}, M}(f)\right\|_{2}=o\left(\frac{1}{2^{\frac{3}{2}(k-1)} \sqrt{M+1}}\right)
\end{aligned}
$$

Hence, Theorem 4.1 has been established.

Proof of Theorem 4.2

$$
\begin{aligned}
c_{n, m} & =\int_{\frac{n-1}{2^{k-1}}}^{\frac{n}{2^{k-1}}} f(t) B_{n, m}(t) \mathrm{d} t \\
& =\int_{\frac{n-1}{2^{k-1}}}^{\frac{n}{2^{k-1}}}\left[\left(f(t)-f\left(\frac{n-1}{2^{k-1}}\right)\right] B_{n, m}(t) \mathrm{d} t+f\left(\frac{n-1}{2^{k-1}}\right) \int_{\frac{n-1}{2^{k-1}}}^{\frac{n}{2^{k-1}}} B_{n, m}(t) \mathrm{d} t\right. \\
& =2^{\frac{k-1}{2}} \int_{\frac{n-1}{2^{k-1}}}^{\frac{n}{2^{k-1}}}\left[\left(f(t)-f\left(\frac{n-1}{2^{k-1}}\right)\right] \beta_{m}\left(2^{k-1} t-n+1\right) \mathrm{d} t\right. \\
& =\frac{1}{2^{\frac{k-1}{2}}} \int_{0}^{1}\left[\left(f\left(\frac{u+n-1}{2^{k-1}}\right)-f\left(\frac{n-1}{\left.\left.2^{k-1}\right)\right]} \beta_{m}(u) \mathrm{d} u, \quad\left(\operatorname{putting} 2^{k-1} t-n+1=u\right)\right.\right.\right. \\
& =\frac{1}{(m+1) 2^{\frac{k-1}{2}}} \int_{0}^{1}\left(\frac{u}{2^{k-1}}\right)^{\alpha} \beta_{m+1}^{\prime}(u) \mathrm{d} u \\
& =\frac{1}{(m+1) 2^{\left(\alpha+\frac{1}{2}\right)(k-1)}} \beta_{m+1}(1)-\frac{1}{(\alpha+1) \sqrt{2 \alpha+3}} \leq \frac{1}{(m+1) 2^{\left(\alpha+\frac{1}{2}\right)(k-1)}} \\
\left\|f-S_{2^{k-1}, M}(f)\right\|_{2}^{2} & =\sum_{n=1}^{2^{k-1}} \sum_{m=M}^{\infty}\left|c_{n, m}\right|^{2} \leq \sum_{n=1}^{2^{k-1}} \sum_{m=M}^{\infty} \frac{1}{(m+1)^{2} 2^{(2 \alpha+1)(k-1)}}, \quad \text { (by Lemma 4.1) } \\
& =\frac{1}{2^{2 \alpha(k-1)}} \sum_{m=M}^{\infty} \frac{1}{(m+1)^{2}}=\frac{1}{(M+1) 2^{2 \alpha(k-1)}} \\
& E_{2^{k-1}, M}^{(2)}(f)=\left\|f-S_{2^{k-1}, M}(f)\right\|_{2}=o\left(\frac{1}{\sqrt{M+1} 2^{(k-1) \alpha}}\right)
\end{aligned}
$$

Thus, Theorem 4.2 has been established.

\section{Method of solution}

5.1 Solution of linear Volterra integral equation of second kind

Consider the linear Volterra integral equation of second kind given by eq ${ }^{n}$ (1.1). Using eq ${ }^{n}$ (3.4), wehave 


$$
\begin{aligned}
& \int_{0}^{x} K(x, t) y(t) \mathrm{d} t= \int_{0}^{x} B^{T}(x) \mathbf{K} B(t) B^{T}(t) Y \mathrm{~d} t=B^{T}(x) \mathbf{K} \int_{0}^{x} B(t) B^{T}(t) Y \mathrm{~d} t \\
&= B^{T}(x) \mathbf{K} \int_{0}^{x} \tilde{Y} B(t) \mathrm{d} t=B^{T}(x) \mathbf{K} \tilde{Y} P B(x) \\
& \text { Then } y(x)=f(x)+B^{T}(x) \mathbf{K} \tilde{Y} P B(x)
\end{aligned}
$$

By evaluating the $\mathrm{eq}^{n}(5.1)$ at $2^{k-1} M$ points $\left\{x_{i}\right\}_{i=1}^{2^{k-1} M}$ in the intervals, we have the following system of linear equations:

$$
B^{T}\left(x_{i}\right) Y=f\left(x_{i}\right)+B^{T}\left(x_{i}\right) \mathbf{K} \tilde{Y} P B\left(x_{i}\right)
$$

Solving the system given by $\mathrm{eq}^{n}$ (5.2), we can find $\mathrm{Y}$ and hence the solution $\mathrm{y}(\mathrm{x})$.

\subsection{Solution of nonlinear Volterra integral equation of second kind}

Consider the Non-linear Volterra integral equation of second kind given by eq ${ }^{n}$ (1.2). Using the Bernoulli wavelet approximation, we have

$$
B^{T}(x) Y=f(x)+\int_{0}^{x} k(x, t) F\left(B^{T}(t) Y\right) \mathrm{d} t
$$

Hence, we have following system of $2^{k-1} M$ equations:

$$
B^{T}\left(x_{i}\right) Y=f\left(x_{i}\right)+\int_{0}^{x_{i}} k\left(x_{i}, t\right) F\left(B^{T}(t) Y\right) \mathrm{d} t
$$

Now, transform the integration in the interval $\left[0, x_{i}\right]$ as $t=\frac{x_{i}(u+1)}{2}$ and

$$
H\left(x_{i}, t\right)=k\left(x_{i}, t\right) F\left(B^{T}(t) Y\right)
$$

Using these, $\mathrm{Eq}^{n}$ (5.6) becomes

$$
B^{T}\left(x_{i}\right) Y=f\left(x_{i}\right)+\frac{x_{i}}{2} \int_{-1}^{1} H\left(x_{i}, \frac{x_{i}(u+1)}{2}\right) \mathrm{d} t
$$

This is the nonlinear system of algebraic equations which can be solved by Newton's method. After obtaining the coefficients, we can find the solution of the integral equation (1.2).

\section{Numerical examples}

Some linear and nonlinear Volterra integral equations have been solved by Bernoulli wavelet technique discussed in this paper and are compared with their exact solutions. The graphs of these solutions are plotted. It is observed that exact solution and approximate solutions of Volterra integral equations are almost equal.

\subsection{Example 1}

Consider the linear Volterra integral equation

$$
y(x)=2 x^{3}-x^{2}-e^{-x^{2}}+1+\int_{0}^{x} e^{-x^{2}+t^{2}} y(t) \mathrm{d} t
$$

$y(x)=2 x^{3}$ is the exact solution of the integral equation (6.1).

Table 1 shows a numerical comparison between exact solution and approximate solutions obtained by Bernoulli wavelet technique. In addition, Fig. 2 shows a graphical representation of exact and approximate solution of linear Volterra integral equation (6.1). 
Table 1 Numerical comparison between Bernoulli wavelet solution and exact solution of example 6.1 for different values of $x$.

\begin{tabular}{|c|c|c|c|c|}
\hline$x$ & Exact Sol $^{n}$ & $\begin{array}{l}\text { Bernoulli wavelet } \\
\mathrm{Sol}^{n}: k=2, m=2\end{array}$ & $\begin{array}{l}\text { Bernoulli wavelet } \\
\mathrm{Sol}^{n}: k=2, m=3\end{array}$ & $\begin{array}{l}\text { Bernoulli wavelet } \\
\text { Sol }^{n}: k=3, m=3\end{array}$ \\
\hline 0 & 0 & 0.0015 & 0.0009 & 0.0011 \\
\hline 0.1 & 0.0020 & 0.0029 & 0.0035 & 0.0020 \\
\hline 0.2 & 0.0160 & 0.0221 & 0.0109 & 0.0160 \\
\hline 0.3 & 0.0540 & 0.0612 & 0.0405 & 0.0537 \\
\hline 0.4 & 0.1280 & 0.1531 & 0.0945 & 0.1300 \\
\hline 0.5 & 0.2500 & 0.3051 & 0.3072 & 0.2548 \\
\hline 0.6 & 0.4320 & 0.4512 & 0.5960 & 0.4479 \\
\hline 0.7 & 0.6860 & 0.0714 & 0.8809 & 0.7313 \\
\hline 0.8 & 1.0240 & 0.8254 & 0.9800 & 0.9719 \\
\hline 0.9 & 1.4580 & 1.3025 & 1.3881 & 1.5324 \\
\hline 1 & 2 & 1.6025 & 1.6502 & 2.0157 \\
\hline
\end{tabular}

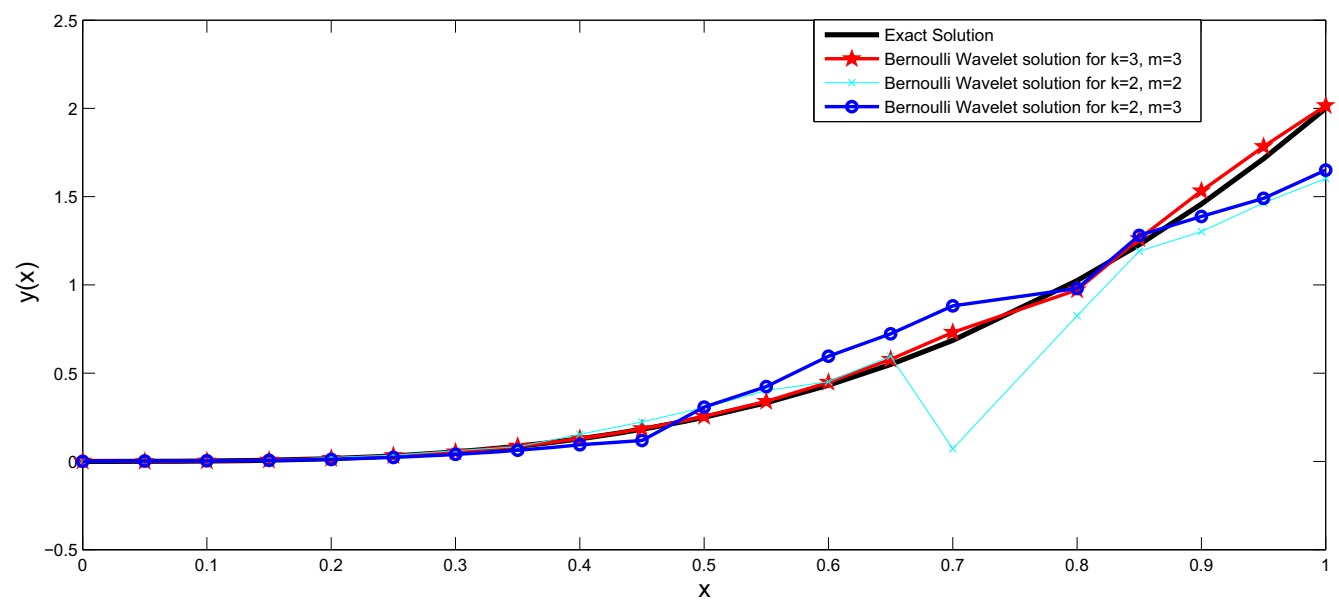

Absolute error

\begin{tabular}{|c|c|c|c|}
\hline $\mathrm{x}$ & For $\mathrm{k}=2, \mathrm{~m}=2$ & For $\mathrm{k}=2, \mathrm{~m}=3$ & For $\mathrm{k}=3, \mathrm{~m}=3$ \\
\hline 0 & 0.0015 & 0.0009 & 0.0011 \\
0.1 & 0.0009 & 0.0015 & 0.0001 \\
0.2 & 0.0061 & 0.0051 & 0.0004 \\
0.3 & 0.0072 & 0.0135 & 0.0003 \\
0.4 & 0.0251 & 0.0335 & 0.0020 \\
0.5 & 0.0551 & 0.0572 & 0.0048 \\
0.6 & 0.0192 & 0.1640 & 0.0159 \\
0.7 & 0.6146 & 0.1949 & 0.0453 \\
0.8 & 0.1986 & 0.0440 & 0.0521 \\
0.9 & 0.1555 & 0.0699 & 0.0744 \\
1 & 0.3975 & 0.3498 & 0.0157 \\
\hline
\end{tabular}

Fig. 2 Comparison of Bernoulli wavelet solution and exact solution of example 6.1.

\subsection{Example 2}

Consider another linear Volterra integral equation given by

$$
y(x)=\frac{x^{2} e^{x}}{\pi} \cos (\pi x)+e^{x}\left(1-\frac{x}{\pi^{2}}\right) \sin (\pi x)+\int_{0}^{x} x t e^{x-t} y(t) \mathrm{d} t
$$

$y(x)=e^{x} \sin (\pi x)$ is the exact solution of the integral equation (6.2). 
Table 2 Numerical comparison between Bernoulli wavelet solution and exact solution of example 6.2 for different values of $x$.

\begin{tabular}{lllll}
\hline$x$ & Exact Sol $^{n}$ & $\begin{array}{l}\text { Bernoulli wavelet } \\
\mathrm{Sol}^{n}: k=2, m=2\end{array}$ & $\begin{array}{l}\text { Bernoulli wavelet } \\
\mathrm{Sol}^{n}: k=2, m=3\end{array}$ & $\begin{array}{l}\text { Bernoulli wavelet } \\
\mathrm{Sol}^{n}: k=3, m=3\end{array}$ \\
\hline 0 & 0 & 0.0101 & 0.0012 & -0.0058 \\
0.1 & 0.3415 & 0.4255 & 0.3902 & 0.3424 \\
0.2 & 0.7179 & 0.7545 & 0.7226 & 0.7173 \\
0.3 & 1.0921 & 1.1902 & 1.1150 & 1.0912 \\
0.4 & 1.4188 & 1.5166 & 1.4555 & 1.4112 \\
0.5 & 1.6487 & 1.7823 & 1.7251 & 1.7068 \\
0.6 & 1.7329 & 1.7703 & 1.8085 & 1.7723 \\
0.7 & 1.6292 & 1.5892 & 1.5662 & 1.6114 \\
0.8 & 1.3081 & 1.2456 & 1.1158 & 0.3105 \\
0.9 & 0.7601 & 0.8278 & 0.5226 & -0.3368 \\
1 & 0 & 0.3636 & -0.2051 & \\
\hline
\end{tabular}

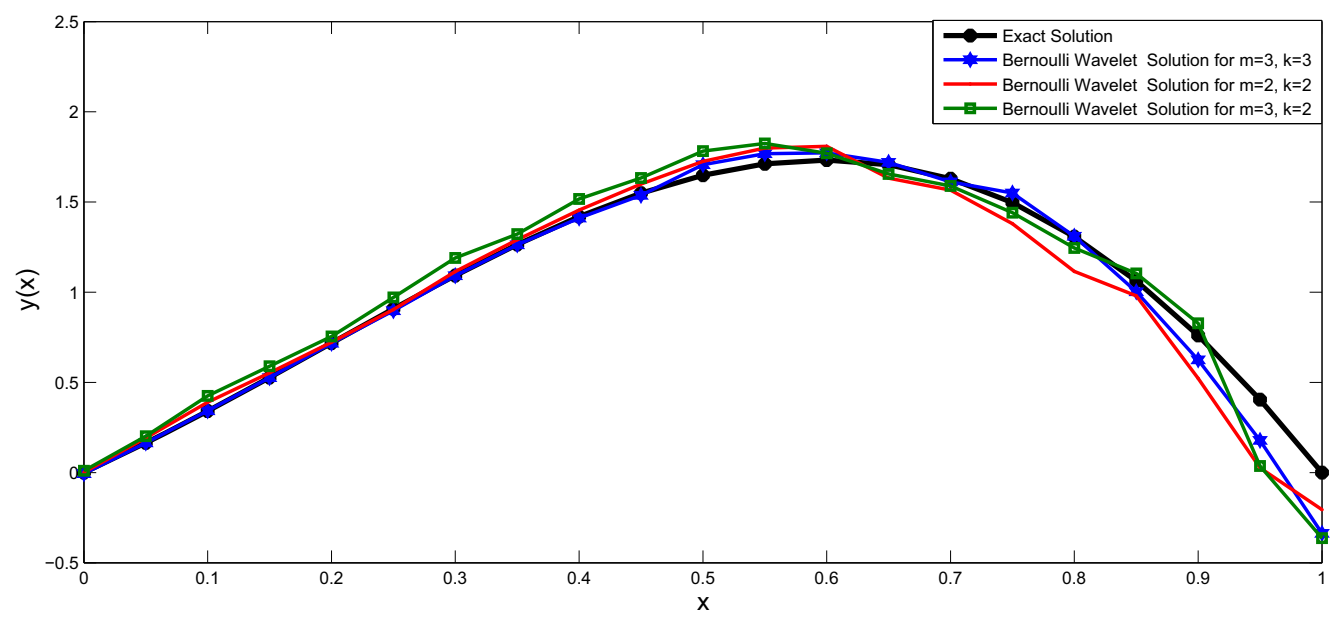

Absolute error

\begin{tabular}{|c|c|c|c|}
\hline $\mathrm{x}$ & For $\mathrm{k}=2, \mathrm{~m}=2$ & For $\mathrm{k}=2, \mathrm{~m}=3$ & For $\mathrm{k}=3, \mathrm{~m}=3$ \\
\hline 0 & 0.0101 & 0.0012 & 0.0058 \\
0.1 & 0.0840 & 0.0487 & 0.0009 \\
0.2 & 0.0366 & 0.0047 & 0.0006 \\
0.3 & 0.0981 & 0.0229 & 0.0009 \\
0.4 & 0.0978 & 0.0367 & 0.0076 \\
0.5 & 0.1336 & 0.0764 & 0.0581 \\
0.6 & 0.0374 & 0.0756 & 0.0394 \\
0.7 & 0.0400 & 0.0630 & 0.0178 \\
0.8 & 0.0625 & 0.1923 & 0.0024 \\
0.9 & 0.0677 & 0.2375 & 0.1354 \\
1 & 0.3636 & 0.2051 & 0.3368 \\
\hline
\end{tabular}

Fig. 3 Comparison of Bernoulli wavelet solution and exact solution of example 6.2.

Table 2 shows a numerical comparison between exact solution and approximate solution obtained by Bernoulli wavelet technique. In addition, Fig. 3 shows a graphical representation of exact and approximate solution of linear Volterra integral equation (6.2). 
Table 3 Numerical comparison between Bernoulli wavelet solution and exact solution of example 6.3 for different values of $x$.

\begin{tabular}{lllll}
\hline $\mathrm{x}$ & Exact Sol $^{n}$ & $\begin{array}{l}\text { Bernoulli wavelet } \\
\mathrm{Sol}^{n}: k=2, m=2\end{array}$ & $\begin{array}{l}\text { Bernoulli wavelet } \\
\mathrm{Sol}^{n}: k=2, m=3\end{array}$ & $\begin{array}{l}\text { Bernoulli wavelet } \\
\mathrm{Sol}^{n}: k=3, m=3\end{array}$ \\
\hline 0 & 0 & -0.01241 & -0.0042 & 0.0021 \\
0.1 & 0.0100 & 0.0091 & 0.0052 & 0.0130 \\
0.2 & 0.0400 & 0.0263 & 0.0290 & 0.0450 \\
0.3 & 0.0900 & 0.0612 & 0.0699 & 0.0955 \\
0.4 & 0.1600 & 0.1004 & 0.1400 & 0.1721 \\
0.5 & 0.2500 & 0.1862 & 0.2096 & 0.2661 \\
0.6 & 0.3600 & 0.2736 & 0.3006 & 0.3748 \\
0.7 & 0.4900 & 0.3599 & 0.3911 & 0.5332 \\
0.8 & 0.6400 & 0.4508 & 0.5010 & 0.6658 \\
0.9 & 0.8100 & 0.5909 & 0.6436 & 0.8393 \\
1 & 1 & 0.7542 & 0.7900 & 1.3390 \\
\hline
\end{tabular}

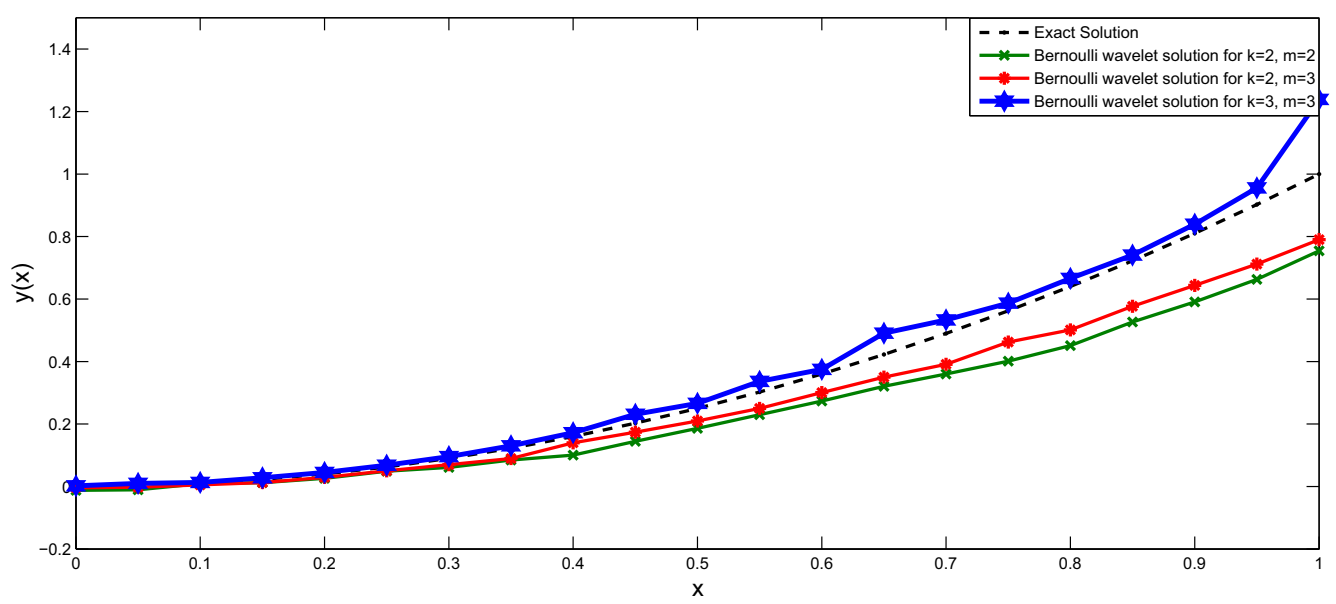

Absolute error

\begin{tabular}{|c|c|c|c|}
\hline $\mathrm{x}$ & For $\mathrm{k}=2, \mathrm{~m}=2$ & For $\mathrm{k}=2, \mathrm{~m}=3$ & For $\mathrm{k}=3, \mathrm{~m}=3$ \\
\hline 0 & 0.0124 & 0.0042 & 0.0021 \\
0.1 & 0.0009 & 0.0048 & 0.0030 \\
0.2 & 0.0137 & 0.0110 & 0.0050 \\
0.3 & 0.0288 & 0.0201 & 0.0055 \\
0.4 & 0.0596 & 0.0200 & 0.0121 \\
0.5 & 0.0638 & 0.0404 & 0.0161 \\
0.6 & 0.0864 & 0.0594 & 0.0148 \\
0.7 & 0.1301 & 0.0989 & 0.0432 \\
0.8 & 0.1892 & 0.1390 & 0.0258 \\
0.9 & 0.2191 & 0.1664 & 0.0293 \\
1 & 0.2458 & 0.2100 & 0.2390 \\
\hline
\end{tabular}

Fig. 4 Comparison of Bernoulli wavelet solution and exact solution of example 6.3.

\subsection{Example 3}

Consider the following nonlinear Volterra integral equation:

$$
y(x)=x^{2}-\frac{x^{5}}{20}+\int_{0}^{x}(x-t)(y(t))^{3} \mathrm{~d} t
$$

$y(x)=x^{2}$ is its exact solution. 
Table 3 shows a numerical comparison between exact solution and approximate solutions obtained by Bernoulli wavelet technique. In addition, Fig. 4 shows a graphical representation of exact and approximate solutions of nonlinear Volterra integral equation (6.3).

\section{Remarks}

1. From Theorem 4.1, we have

$$
E_{2^{k-1}, M}^{(1)}(f)= \begin{cases}o\left(\frac{1}{2^{\frac{1}{2}(k-1)}}\right), & \text { if } M=0 \\ o\left(\frac{1}{2^{\frac{3}{2}(k-1)} \sqrt{M+1}}\right), & \text { if } M \geq 1 .\end{cases}
$$

hence $E_{2^{k-1}, M}^{(1)}(f) \rightarrow 0$ as $M \rightarrow \infty, k \rightarrow \infty$.

2. From Theorem 4.2 , we have

$$
E_{2^{k-1}, M}^{(2)}(f)=o\left(\frac{1}{\sqrt{M+1} 2^{(k-1) \alpha}}\right) \rightarrow 0 \text { as } M \rightarrow \infty, k \rightarrow \infty \text {. }
$$

Therefore, $E_{2^{k-1}, M}^{(1)}(f)$ and $E_{2^{k-1}, M}^{(2)}(f)$ are the best possible estimators in wavelet analysis (Zygmund [22]).

3. Solution of linear and nonlinear Volterra integral equations, obtained by Bernoulli wavelet technique are compared with their exact solution with the help of tables and graphs. In addition, we observe that approximate solution and exact solution are almost same. This is a significant achievement of this research paper.

4. From the graphs of the solution, we observe that when we increase the values of $\mathrm{k}$ and $\mathrm{m}$, the approximate solutions are more close to the exact solution.

Acknowledgements Shyam Lal, one of the authors, is thankful to DST-CIMS for encouragement to this work. Satish Kumar, one of the authors, is grateful to C.S.I.R. (India) for providing financial assistance in the form of Junior Research Fellowship vide Ref. No. 17/12/2017 (ii) EU-V Dated:13-02-2019 for his research work. The authors are grateful to the referee for his valuable comments and suggestions, to improve the quality of this research paper.

Open Access This article is licensed under a Creative Commons Attribution 4.0 International License, which permits use, sharing, adaptation, distribution and reproduction in any medium or format, as long as you give appropriate credit to the original author(s) and the source, provide a link to the Creative Commons licence, and indicate if changes were made. The images or other third party material in this article are included in the article's Creative Commons licence, unless indicated otherwise in a credit line to the material. If material is not included in the article's Creative Commons licence and your intended use is not permitted by statutory regulation or exceeds the permitted use, you will need to obtain permission directly from the copyright holder. To view a copy of this licence, visit http://creativecommons.org/licenses/by/4.0/.

\section{Declarations}

Conflict of interest The first author declares that he has no conflict of interest. The second author declares that he has no conflict of interest.

Ethical approval This article does not contain any studies with human participants or animals performed by any of the authors.

\section{References}

1. Jumah, A.: Numerical solution of Volterra integral equation of second kind. Int. J. Comput. Sci. Mobile Comput. 2016, 509-514 (2016)

2. Kouba, O.: Lecture notes: Bernoulli polynomials and applications. arXiv:1309.7560v1 [mah.CA] (2013)

3. Keshavarz, E.; Ordokhani, Y.; Razzaghi, M.: Bernoulli wavelet operational matrix of fractional order integration and its applications in solving the fractional order differential equations. App. Math. Model. 38, 6038-6051 (2014)

4. Keshavar, R.: The Bernoulli wavelet Operational matrix of integration and its application in the solution of linear and nonlinear problems in calculus of variations. Appl. Math. Comput. 351, 83-98 (2019)

5. Lu, D.-Q.: Some properties of Bernoulli polynomials and their generalizations. Appl. Math. Lett. 24, 746-751 (2011)

6. Liang, X.Z.; Liu, M.; Che, X.: Solving second kind integral equations by Galerkin methods with continuous orthogonal wavelets. J. Comput. Appl. Math. 136, 149-161 (2001) 
7. Lepik, Ü.; Tamme, E.: Solution of nonlinear Fredholm integral equations via the Haar wavelet method. Proc. Estonian Acad. Sci. Phys. Math. 56, 17-27 (2007)

8. Maleknejad, K.; Mirzaee, F.: Numerical solution of linear Fredholm integral equations system by rationalized Haar functions method. Int. J. Comput. Math. 80(11), 1397-1405 (2003)

9. Shiralashetti, S.C.; Lamani, L.: Fibonacci wavelet based numerical method for the solution of nonlinear Stratonovich Volterra integral equations. Sci. Afr. 10, e00594 (2020)

10. Shiralashetti, S.C.; Lamani, L.: Numerical solution of stochastic integral equations using CAS wavelets. Malaya J. Mat. 1, 183-186 (2020)

11. Shiralashetti, S.C.; Lamani, L.; Naregal, S.S.: Numerical solution of integral equations using Bernoulli wavelets. Malaya J. Mat. 1, 200-205 (2020)

12. Shiralashetti, S.C.; Lamani, L.: A modern approach based on Bernstein polynomial multiwavelets to solve Fredholm integral and system of Fredholm integral equations. South East Asian J. Math. Math. Sci. 16(3), 251-268 (2020)

13. Shiralashetti, S.C.; Lamani, L.: Bernstein polynomial multiwavelets operational matrices method for the numerical solution of system of linear Stratonovich Volterra integral equations. J. Sci. Res. 65(1), 283-289 (2021)

14. Srivastava, H.M.; Shah, F.A.; Irfan, M.: Generalized wavelet quasilinearization method for solving population growth model of fractional order. Math. Methods Appl. Sci. 43(15), 8753-8762 (2020)

15. Shah, F.A.; Irfan, M.; Nisar, K.S.; Matoog, R.T.; Mahmoud, E.E.: Fibonacci wavelet method for solving time-fractional telegraph equations with Dirichlet boundary conditions. Results Phys. 2021, 104123 (2021). https://doi.org/10.1016/j.rinp. 2021.104123

16. Shah, F.A.; Irfan, M.: Generalized wavelet method for solving nonsteady heat transfer model of fractional order. SeMA J. 2021, 1-16 (2021)

17. Shiralashetti, S.C.; Mundewadi, S.: Bernoulli Wavelet based Numerical Method for the solution of Abel's Integral equations. Int. J. Eng. Sci. Math. 6, 68-70 (2017)

18. Shah, F.A.; Irfan, M.: Generalized wavelet method for solving fractional bioheat transfer model during hyperthermia treatment. Int. J. Wavelets Multiresol. Inf. Process. 2020, 2050090 (2020). https://doi.org/10.1142/S0219691320500903

19. Shah, F.A.; Irfan, M.: Numerical solution of fractional bioheat transfer model under moving heat source using wavelet method. Num. Com. Meth. Sci. Eng. 2(1), 1-9 (2020)

20. Yousefi, S.; Banifatemi, A.: Numerical solution of Fredholm integral equations by using CAS wavelets. Appl. Math. Comput. 183, 458-463 (2006)

21. Zheng, X.; Wei, Z.: Estimates of approximation error by Legendre wavelet. Appl. Math. 7, 694-700 (2016)

22. Zygmund, A.: Trigonometric Series, vol. I. Cambridge University Press, Cambridge (1959)

Publisher's Note Springer Nature remains neutral with regard to jurisdictional claims in published maps and institutional affiliations. 